\title{
Diversity or Disparity? The Concentration of Funded Research Topics in the United Kingdom
}

\author{
Emil Bargmann Madsen ${ }^{1 \dagger}$
}

March 2, 2020

\begin{abstract}
The misalignment between societal needs and the priorities of conducted research have spurred calls for more attention to what types of research is actually funded. At the same time, published research exhibits strong path dependency by becoming more focused on well-established and reoccurring topics. The distribution of attention over various topics reflect the broader political economy and power structures in science, but whether path dependency starts already at the funding stage is largely unknown. In this article, I ask how skewed and path dependent the distribution of research funding is with respect to research topics. Using data on public funding of more than 30,000 research projects in the United Kingdom since 2006, I show that competitive public research funding is consistently concentrated on a minority of topics. These privileged topics continue to attract the majority of funding over time, and generally stay within the top parts of the funding distribution. Funding of research topics thus seems to follow a process of cumulative advantage, but while higher prior funding levels predicts higher subsequent funding, there is a decreasing marginal return.
\end{abstract}

Keywords: research funding, funding concentration, Matthew effect, topic diversity, Bayesian modelling

JEL codes: I23, O31, O32, O38

\section{Introduction}

The current system for allocating research funding is often described as facing a "prolonged crisis" (Fang and Casadevall 2016) or "systematic flaws" (Alberts et al. 2014). The problems underlying these descriptions are many, but often include a growing concern over falling success rates and concentration of funding in the hands of the few (Fortin and Currie 2013; K. Gross and Bergstrom 2018; Peifer 2017; Vaesen and Katzav 2017; Wahls 2018b). These concerns have largely been corroborated empirically. A wealth of research demonstrate cumulative advantage or "Matthew Effects", where narrow groups of researchers and research organisations accumulate disproportionate amounts of research funding.

\footnotetext{
${ }^{1}$ Danish Centre for Studies in Research and Research Policy, Department of Political Science, Aarhus University, email: ebm@ps.au.dk

† I am indebted to Kaare Aagaard, Carsten Jensen, Jens Peter Andersen, Michael Hopkins, Frederique Boné, Danielle Rotolo, Jesper W. Schneider, Andreas Kjær Stage, and participants at both the $25^{\text {th }}$ Annual SPRU Forum and the Annual Internal CFA PhD Seminar for considerable input, advice and comments, which helped improve this paper. All remaining errors are my own.
} 
Funding distributions are extremely skewed (Katz and Matter 2019; Larivière et al. 2010, 49; Ma, Mondragón, and Latora 2015, 14761; Mongeon et al. 2016; Peifer 2017; Wahls 2018b, 2018a) and individuals in the top of the distribution tend to accumulate more funding compared to the bottom over time (Jacob and Lefgren 2011; Katz and Matter 2019; Lerchenmueller and Sorenson 2018).

However, the focus on individual Matthew effects have neglected that inefficiencies in science funding may be most pressing at the research topic level. The distribution of funding to different research topics and problems is important because it indicates how a society values different types of scientific progress and research strategies (Klavans and Boyack 2017b). Studies have documented widespread disparities between societal needs and research priorities (Evans, Shim, and Ioannidis 2014; C. Gross, Anderson, and Powe 1999; Manton et al. 2009; Yao et al. 2015; Yegros-Yegros et al. 2018). The biomedical sciences experience marked stratification in funding of disease-specific research (Evans, Shim, and Ioannidis 2014; Head et al. 2013, 2015; Yao et al. 2015). Funding is also skewed towards translational biomedicine and pharmaceuticals, while neglecting public health and prevention studies (Head et al. 2016, 184; Jones and Wilsdon 2018, 15-18). This tendency is likely not endemic to the medical sciences. Similar concerns have been aired from researchers in physics, where directed funding to nanotechnology, energy materials, and photonics may crowd out funding for alternative basic research (Nature 2003). Contrary to Matthew Effects for individual researchers, we are still somewhat amiss regarding the distribution of funding across research topics. The tendency for funding to concentrate in few research topics is often postulated (Alberts et al. 2014; Fortunato et al. 2018), but seldom put to the test. A recent exception, focusing on US funding 2008-2013, have nevertheless documented a considerably skewed distribution of funding over topics (Klavans and Boyack 2017a). The concentration of funding correlate well with the prominence of topics in the scholarly literature but also the amount of funding a topic has previously attained (Klavans and Boyack 2017a, 1167-69). Prominent topics accumulate funding while non-prominent topics are increasingly disadvantaged over time. However, quantitative assessments of funding distributions are often focused on more limited research disciplines (Head et al. 2013, 2015; Hoonlor, Szymanski, and Zaki 2013), the US or sub-national contexts (Best 2012; Bromham, Dinnage, and Hua 2016; Klavans and Boyack 2017a; Manton et al. 2009; Mongeon et al. 2016; Sampat 2012; Yao et al. 2015), and limited time periods (Klavans and Boyack 2017a). I expand upon these studies by presenting data on 30,662 individual grants awarded by public research councils in the United Kingdom from 2006 until 2017. I aim to provide a broad description of the extent that research funding is concentrated in research topics and how concentration have changed over time by focusing on a diverse set of disciplines, and a much longer time period than previously considered.

I proceed in four steps. First, I summarise the empirical efforts to study funding concentration for individuals and argue for why we should start considering similar dynamics for research topics. Second, I outline a procedure for linking grants to topics through analysis of publications by the grantees. Third, I present evidence for a concentration of funding in topics over time, and how funding distributions become static. Lastly, I discuss some possible drivers of cumulative advantage for topics. 


\section{Empirical perspectives on funding and topic concentration}

Examinations of how scholarly attention is distributed have largely followed two separate tracks. Firstly, researchers have focused on how certain individuals and institutions attract disproportionately large amounts of grants and resources. Secondly, a growing orthodoxy have been documented in different scientific disciplines where the same research questions are seemingly re-examined again and again at an increasing rate. Few studies have however joined these perspectives. This is important as these two processes are likely not independent. The concentration of funding in individuals and organisations likely privileges the topics studied by these. Conversely, a concentration in topics may give unequal funding opportunities for researchers acting in different fields and drive cumulative advantage at the individual level.

I start out by reviewing the empirical evidence of cumulative advantage in funding, pointing to three recurring patterns: Extreme skew in funding amounts and number of grants, stasis or path dependency of unequal funding distributions over time, and tendency for prestige to drive concentration. Next, I argue that these tendencies may carry over to concentration in topics funded.

\subsection{Cumulative advantage in research funding}

Early accounts from sociologists of science argued that competition for recognition between autonomous scientists would secure an efficient allocation of funds and efforts among scientific problems (Hagstrom 1974, 1-2; Merton 1957; Polanyi 1962). This view casts the scientific community as a self-regulating system effectively distributing attention across research problems (Sarewitz and Pielke 2007, 7). Robert Merton's work on the Matthew Effect seems to break with such a view. In Merton's characterisation of the allocation of rewards in science, recognition accrues to those who provide original contributions to science (Merton 1957, 639). However, even though such contributions receive accolades, peer-recognition is often distributed with a gradient (Cole and Cole 1967, 382; Merton $1968,56)$. Well-established scholars tend to accumulate recognition through publication, awards, and grants to a much higher degree than less well-known researchers, irrespective of talent or the intrinsic merit of their contributions (Bol, de Vaan, and van de Rijt 2018, 1; Goldstone 1979, 385). This process is self-reinforcing as recognition signals competence to those who distribute scientific resources (Reskin 1977, 493; Zuckerman 1967, 396). While Merton himself did not undertake any formal analysis, his hypothesis of cumulative advantage for individuals and organisations (Merton 1988, 606) became the focus of almost all later empirical investigations. Early studies focused on the concentration of publications and citations of individual researchers (Allison, Long, and Krauze 1982; Allison and Stewart 1974; Cole and Cole 1967; Long 1978; Reskin 1977), but recognition through grants have lately become 
a strong focus in the literature. Overall, studies document three common tendencies in competitive grant competitions across funding bodies and countries: an extreme skew of cumulative funding distributions, wide-spread stasis, or path dependency, in who receives funding, and a strong influence of prestige at both the institutional and individual level.

Extreme skew. Nearly all studies find a significantly skewed distribution of research funds, in terms of both number of grants and amount of funding, across individuals and research institutions. A large proportion of studies rely on funding data from the National Institutes of Health (NIH), and typically find that $20 \%$ of funded researchers receive around $50 \%$ of funding amounts, while $10 \%$ of research institutions accumulate $80 \%$ of funding (Katz and Matter 2017, 4-5; Peifer 2017; Wahls 2018b, 2018a). Outside the US and NIH, the skew appears even more extreme. In Quebec, around 20-25\% of researchers in natural sciences received $80 \%$ of external funds (Larivière et al. 2010; Mongeon et al. 2016), while 10$15 \%$ of researchers accumulated $80 \%$ of funds in the biomedical sciences (Larivière et al. 2010, 49). Funding from the Engineering and Physical Sciences Research Council (EPSRC) in the UK appear to be exhibit the most skew. From 1985 to 2013, 8 \% of Principal Investigators (PIs) received half of all funding, while $90 \%$ of funding was awarded to just $20 \%$ of universities (Ma, Mondragón, and Latora 2015, 14761)

Stasis. Besides a varying, but generally, large skew of grants and funding amounts, a number of studies also show a deepening of these inequalities over time. Researchers funded by the NIH generally accumulate much more funding that non-winners with similar grant review scores. Some estimates show that R01 grantees accumulated an average of $\$ 227,000$ five years after their initial grant, and $\$ 648,000$ after six to ten years, and early-career grant recipients also have much larger propensities for receiving a mid-career grant up to 8 years after an initial grant (Jacob and Lefgren 2011, 1173-74; Lerchenmueller and Sorenson 2018, 1012). Similar effects have also been observed for prestigious Veni grants in the Netherlands. Grant winners, with review scores similar to high-ranking non-winners, had accumulated an average of $€ 40,000$ more within 4 years of receiving a grant. After 8 years, this gap had widened to $€ 180,000$ (Bol, de Vaan, and van de Rijt 2018, 3). Individual grant winners in the NIH also showed a considerable stasis when it came to winning further NIH grants. Grant winners entering an 8 year period in the top $10 \%$ of the funding distribution also had a $58 \%$ chance of staying in the top ranked percentile (Katz and Matter 2019, 14). These individual feedback effects also seem to manifest themselves across the whole system. In the NIH, the Gini coefficient for both individuals rose from 0.4 in 1985 to 0.5 in 2010, and from 0.81 to 0.86 for research organisations (Katz and Matter 2019, 9). For the EPSRC, Gini coefficients rose from below 0.5 to nearly 0.7 in the same period, with inequality between universities being consistently higher than between PIs (Ma, Mondragón, and Latora 2015, 14761).

Prestige of institutions and individuals. One persistent factor of stratification in studies of funding distributions have been the prestige assigned to individuals and organisations. Prestigious US universities are 1.7 times as likely to get funding and to get one application funded each year compared 
to less prestigious institutions. The amount of funding for investigators from prestigious universities were on average 2.4 times higher and the mean annual award size was 1.5 times higher (Wahls 2018a, 3-4). This prestige effect may also give funded PI's an edge in the publication system, as journals with higher impact factors tend to publish NIH funded investigators with higher median funding levels (Katz and Matter 2017, 11). In general, project funding schemes seem to be subject to strong gatekeeper functions. Prestigious research institutions not only accumulate more funding, but also impact the amount of funding awarded to others. For the EPSRC, Ma et al. show (Ma, Mondragón, and Latora 2015) that UK universities form funding 'rich clubs' where “... affiliations with a high number of grants tended preferentially to collaborate among each other, forming tightly interconnected communities...” (Ma, Mondragón, and Latora 2015, 14762). Whether individual researchers serve similar gatekeeper roles is still questionable. In the Canadian Research Councils, researchers who are centrally placed in coauthorship networks also attract more funding (Ebadi and Schiffauerova 2015, 12).

\subsection{Concentration of attention and funding in research topics}

Despite the focus on cumulative advantage for individuals and institutions in the extant literature, the idea that topics also accumulate grants in a differentiated manner is not new. Early studies of Matthew Effects already speculated that cumulative advantage for individual scientists might carry over to the type of topics these considered. A researcher occupying a central location in a research area retain productivity advantages. Established researchers tend to work on synthesizing or integrating research topics, and are able to build up 'cultural capital' that can be transferred into resources (Allison and Stewart 1974; Hargens, Mullins, and Hecht 1980, 68-69). Whether this is actually the case is still not clear. Recent studies suggest that the extreme skew and stasis of funding also affects the distribution of funding to topics. For example, 50-75 \% of projects funded by the NIH relate to $5 \%$ of human proteincoding genes. The likelihood of becoming a PI is much lower for researchers studying the least studied genes compared to those who study the most studied (10\% vs. 20-25\%) (Stoeger et al. 2018, 8). Similarly, $86 \%$ of articles indexed in MEDLINE investigated pairs of chemical substances previously investigated, while $14 \%$ investigated completely new chemical entities or new relationships between known substances (Foster, Rzhetsky, and Evans 2015, 886). Researchers' reliance on competitive funding have grown in recent years, and in the same period these researchers have become more conservative in the topics they explore, with more repeated topic combinations (Rzhetsky et al. 2015, 14570-71).

However, the connection between funding concentration for individual researchers and for topics may also run counter. There may also be a feedback in funding success from topics to individuals. Career advancement in relation to grants have been speculated to demand a steady production of publications through incremental contributions to the same research agenda. Researchers rarely make radical shift to an entirely new set of research topics, perhaps because demonstrating continuity and cumulative scholarship is the best career path for a researcher (Jia, Wang, and Szymanski 2017, 1). Studies of this 
are still scarce. In computer science, a $10 \%$ increase in the number of published papers in a given topic is followed by a $75 \%$ probability of an increase in the number of NSF grants awarded on the same topic (Hoonlor, Szymanski, and Zaki 2013, 80). So far, Klavans and Boyack (2017a) have provided the broadest examination of topic and funding concentrations. They examine how topic prominence in publications correlates with later funding by using data from 314,095 federal research grants from 2008-2014. ${ }^{2}$ Calculating text similarity between grant titles and abstracts with publications in the Scopus database, they assigned the most similar topic to each grant (Klavans and Boyack 2017a, 1167). They find that topics in scholarly articles differ trough their prominence (i.e. how much the topics are cited and viewed) (Klavans and Boyack 2017a, 1165). How intensely a topic is funded is then both a function of its historical funding levels, and the prominence of that topic. Funding to a topic correlates extremely well with funding levels in earlier time periods and the historical prominence of a topic, with $73 \%$ of the variation in funding levels being explained by these two factors (Klavans and Boyack 2017a, 1168-69). Only 3.7\% of topics with low prominence received funding, while the 136 topics with the highest prominence, had an average of 184 U.S. authors, who received an average of $\$ 91,870$ each (Klavans and Boyack 2017a, 1168-69). Topics are thus very unequal in the attention they receive, receive differentiated levels of research funding, and this inequality seems to persist over time. This seems to mirror the three central tendencies described above. Funding to topics is skewed, static, and if topic prominence in publications reflect prestige, then prestige affect the amount of funding a topic receives. Moreover, topics attracting the bulk of funding in a given period are perhaps the topics currently deemed 'important'. The volume of publications on a topic follow current research policy, where earmarked funding for topics such as nanotechnology or bio-fuels also spark more research in these areas (Domenico, Omodei, and Arenas 2016, 7)

I expand on these points by analysing the distribution and landscape of public funding in the UK through time. The purpose of my investigation is three-fold: (i) To attempt to expand the study of cumulative advantage in science beyond individual researchers and institutions by focusing on cognitive communities arranged around common research topics. (ii) To inductively describe the skewness and stasis in how funding is allocated to research topics, and how funding may cluster in already well-funded and prestigious topic areas. (iii) To discuss how policy factors external to science itself may contribute to funding concentrations.

\section{Materials and Methods}

The analysis relies on a unique database of individual grants awarded to researchers in the United Kingdom covering, as a minimum, the last decade, and 97,861 grants. To assess whether funded topics reflect a process of cumulative advantage in scientific resources, I proceed in three successive steps. First,

\footnotetext{
${ }^{2}$ Covering the NSF, NIH, CDC, NASA, NIFA, and the CDMRP.
} 
I combine automated and manual collection of grant data from seven public research councils, and across a wealth of different research fields. Second, I connect individual grants to a set of probable research topics by utilising fine-grained topic information from 180,100 scholarly publications linked to a grant. Third, I map the spread of funded research topics and discuss the potential mechanisms affecting this spread. It is important to note that my data only documents the distributional outcomes of successful grants. Because I rely on publicly available information on funded grant proposals, I do not assess how the rejection of grant applications play into the cumulative advantage processes of scientific topics. Furthermore, I cannot account for strategic choices to not apply for funding or alternative means of financing research.

\subsection{Data}

To construct the database on individual grants, I used a number of different data sources. For the Research Councils UK (RCUK), I obtained all available funded projects from each of the eight research councils subsumed under the RCUK. ${ }^{3}$ Project-level data were available in the RCUK Gateway to Research Database from 2005 to 2017. I consider only grants with a research focus, and dropped all grants aimed at students, intramural grants, skill development, etc. (See Figure S1 for the sample of grants used). Each funded project is assigned to one principal investigator. In the case of larger centre grant given for the establishment of a research centre of excellence, two choices were made. Centre grants directed towards a certain research organisation (i.e. the PI is a rector, department head, etc.) were left out of the analysis. This ensures that no grant is linked to publications from a PI who might not have been using the grant. For centre grants giving group-leaders or PIs a possibility to create a new research centre, I included the grant.

I matched each principal investigator to their publications using two data sources. First, I used the RCUK Gateway to Research to identify self-reported outcomes of grants, and narrowed the search to only journal articles and reviews. This yielded a gross sample of 305,247 publications directly linked to a specific grant. I then matched these self-reported publications to article-level information through the in-house version of Clarivate Analytics' Web of Science database hosted at the Centre for Science and Technology Studies at Leiden University. The Leiden database is an augmented version of the Web of Science with a thorough author-name disambiguation assigning individual cluster IDs to publications from the same author. This set of PI-linked publication yielded 180,100 documents (See Figure S1 for an overview of the final set of grants).

\footnotetext{
${ }^{3}$ These eight councils are the non-departmental bodies: Engineering and Physical Sciences Research Council (EPRSC), Biotechnology and Biosciences Research Council( BBSRC), Medical Research Council (MRC), Economic and Social Research Councils (ESRC), Arts and Humanities Research Council (AHRC), Natural Environment Research Council (NERC), Science and Technology Facilities Research Council (STFRC), and Innovate UK (formerly the Technology Strategy Board, TSB)
} 


\subsection{Connecting topics and grants}

Science encompasses different communities with researchers intuitively orienting their work towards specific communities or subdivisions of science. These subdivisions could be denoted as fields, specialities, or disciplines (Small and Griffith 1974). Furthermore, some hierarchical partition of these communities is possible: There is broad research fields such as Physics, more specialised, subsumed, disciplines such as Astrophysics or Condensed Matter Physics, and then individual topics within these disciplines. I define a topic as a cognitive space materialised as a collection of documents that focus on a common intellectual interest, such as a research problem (Klavans and Boyack 2017a, 1159), and researchers working on these problems. Scientific documents have often been categorised based on the journal in which they appear, but journals are broad in scope, and may cover many topics, or a topic may be spread across multiple journals. Instead, a document level-classification is needed. Besides the journalbased 250 research areas classified in the Web of Science, the Leiden database offers more detailed article-level classifications through 858 meso-level topic clusters. For each principal investigator, I recorded their distribution of research topics through their published scholarly articles. The topic clusters are based on direct citation links between articles, where each article constitutes a node in a network with direct citations between papers constituting an edge between a pair of nodes. A Smart Local Moving (SLM) algorithm (Waltman and Van Eck 2013) is applied for community detection and clustering into topics. Individual article nodes are moved into a partition and then aggregated so that each topic constitutes a node in an aggregated network. The full procedure is outlined in Waltman and van Eck (2012) and Traag, Waltman, and van Eck (2019). Their method outlines a procedure for constructing a set of micro-level topics or fields, and how these topics can then be aggregated to form a smaller set of meso-level or macro-level topics. An example of the use of micro-level topics is the CWTS Leiden rank of universities where 4535 topics are used. I, instead, rely on the more aggregated 858 mesolevel topics. Because each PI may work on a set of topics, or a grant relates to different areas, I calculated a topic weight for each grant with the most frequent topic in the publication set weighing the most. Using this weight, I split the grant amount and number of grants so to assign the weighted amount of money and grant numbers to a topic by year. The weighing of funding per grant can be illustrated through a simple, fictive example: A grant may be awarded for $£ 100,000$ and result in three publications within two different topics, which we denote topic $A$ and topic $B$. If two of the publications are on topic $A$, and one is on topic $B$, the grant and the grant amount is weighted according to the frequency of these topics. Based on this one grant, topic $A$ achieves 0.66 grants and $£ 66,666.66$, while topic $B$ receives 0.33 grants and $£ 33,333.33$. I then sum up these weighted number of grants and amounts for each topic. This approach differs from other studies, where grant abstracts are compared to publication abstracts, and the most similar abstract topic is assigned to a grant (Klavans and Boyack 2017a). Weighting grants according to multiple topics instead capture either the mono- or interdisciplinary character of a grant, 
and have previously been used for analysing topic diversity in the National Science Foundation (Nichols 2014). Figure 1 provides a topical map of the Research Council funding landscape, based on the outlined weighting method. Each bubble represents one of 858 topics, sized by the weighted amount of funding for each topic in the period 2005 to 2017. The distance between each topic indicates the relatedness of these based on citation links. Even though the algorithmically constructed research topics may ameliorate some problems with journal-based classification, they do carry other problems. For one, the Web of Science coverage of publications from different research areas are still skewed towards the medical- and natural sciences. This may bias what type of grants I am able to link with a publication and topic. Second, the size of research topics in terms of number of publications differ. Observations of skewed funding distributions may then be a result of size differences in the publication input. However, these skewed distributions of papers and funding could also be endogenous to each other. Certain topics may be popular, resulting in both more publications and funding. To try to ensure that my results are robust to these problems, I recreate many of the main results for individual research areas by performing analyses on each research council.

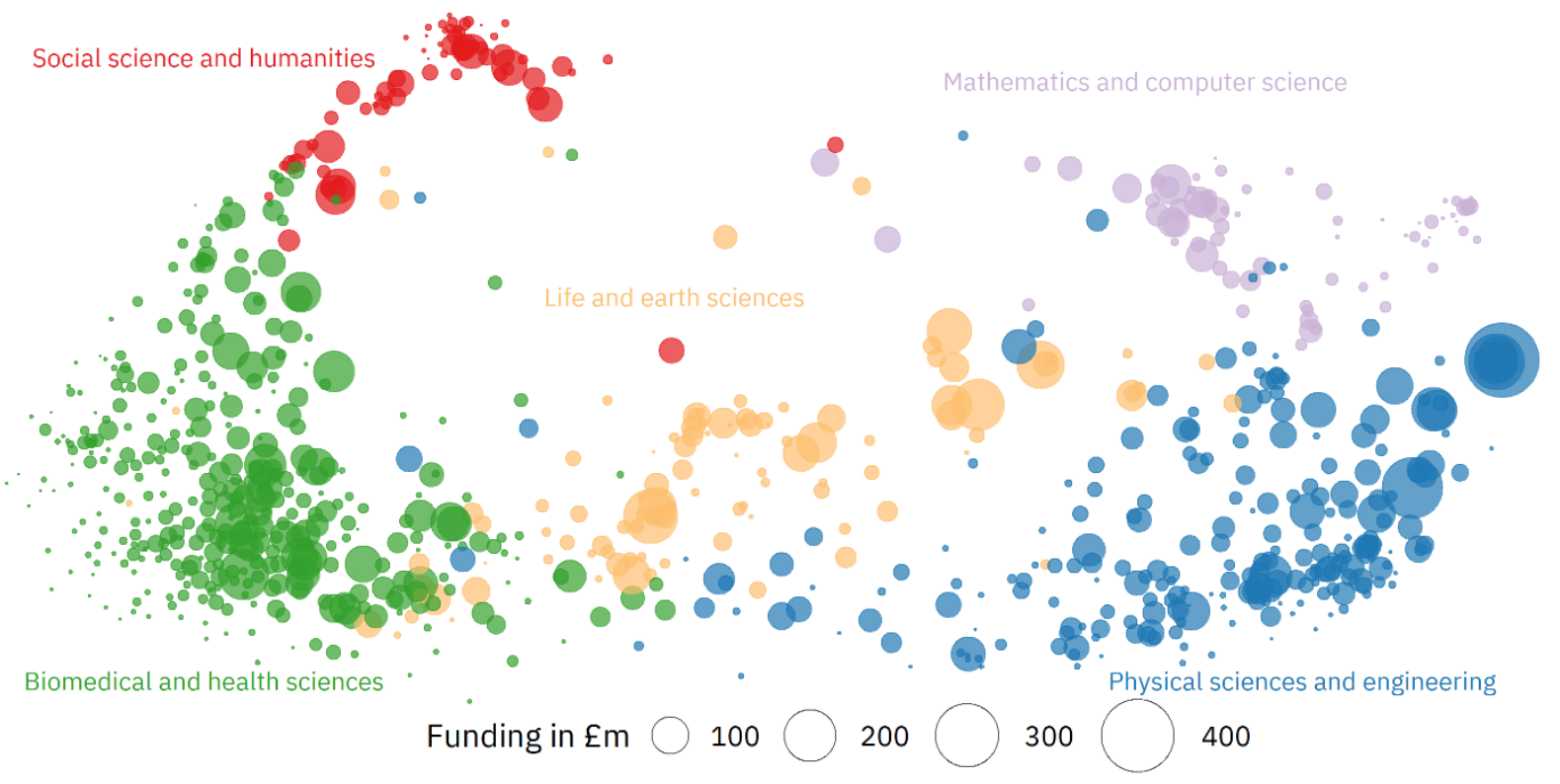

Figure 1. Map of funded topics. Funding amounts for 858 topics between 2005 and 2017.

\subsection{Analytical approach}

To explore how public research funding has been spread across research topics I conduct two sets of analyses. First, I focus on how the distribution of funding have unfolded with respect to the topics actually funded. This part neglects the span of topics not covered by funding from the national research councils but focuses on the inequality in topics actually funded. Given a lack of data on the research topics of grant applications not funded, or the topics focus of researchers who did not apply for funding, 
the analysis likely underestimate the funding inequality between topics. This is essentially a problem of self-selection. Topics with low funding success is not represented and researchers who anticipated low success because of their research topics are not accounted for.

While previous studies have examined inequality at the individual and institutional level (Katz and Matter 2019; Mongeon et al. 2016; Wahls 2018a), I focus on the topic level. Just as investigators participate in a funding 'economy' where grants are the 'income' (Katz and Matter 2019), topics may similarly 'compete' for attention through funding. I calculate the cumulative distribution of funding amounts and the number of grants for all topics researched by funded scientists for the period 20062017. Furthermore, I investigate how the cumulative funding and grants distributions have changed over time. To assess the skewness of these distributions, I calculate three indices of inequality or dispersion of both number of grants and grant amounts. First, I employ one of the most popular indices for measuring income concentration; the Gini coefficient. The Gini coefficient have found widely applied in most studies of inequality in research funding (Katz and Matter 2017; Ma, Mondragón, and Latora 2015) because of its direct relationship with the distributional Lorenz curve: It traces the ratio of the area between a perfect egalitarian Lorenz curve and the empirical curve to the total area under the egalitarian curve. However, the Gini is often criticised for being insufficiently sensitive to changes in the tails of a distribution(Atkinson, Piketty, and Saez 2011, 10). In studies of income inequality, this has led to a greater focus on the share of income in different percentiles of a population, and the ratio of these shares. Here, differences between the very top and bottom have attracted particular attention. I therefore calculate funding and grant shares for percentiles of funded topics, and compute the Palma index of top $10 \%$ funding shares vs. bottom 40 \% funding shares (Cobham, Schlögl, and Sumner 2016).

To investigate the cumulative advantage of topics over time, I attempt to assess the funding trajectory of each topic from the first year it was funded. The year a topic enters the pool of funded topics, it will enter a given percentile rank according the amount of funding allocated to the topic. I then calculate the percentile rank for a topic in all subsequent years, and show how topics that entered the distribution in different rank brackets tend to stay within those brackets.

Finally, I quantify how the funding of topics may exhibit path dependency and positive feedback by estimating a Bayesian hierarchical model. First off, this is complicated by how we measure "success" for a topic across the years of the funding data. In a given year, a topic can attract funding or not, which is in itself a measure of how successful the topic is. Given that it does attract funding, the size of the amount indicates something about the priority placed on that topic in the funding system. These two processes are essentially two linked events that together define the topic priorities in a year. However, a topic can attract zero funding both because no priority is placed on it, but a small amount of funding can also signify low priority. To accommodate this, I treat funding for topics as arising from multiple processes. Priority can be set both through no, or low levels of funding. I incorporate this in a model of path dependency by modelling the amount of funding to a topic as a mixture between a Bernoulli and Gamma process (a hurdle gamma model), i.e. a mixture of zero and positive values. I use past funding 
amounts for a topic to model how static the distribution of funding is. Because early establishment of prestige for some topics may affect the amount of funding for the topic later, I include a series of lagged levels of funding amounts for each topic for a given year. These lags measure the funding amounts for a topic $i$ in $1-5$ years prior. The model is estimated as a hierarchical model with a random intercept for each individual topic. Including a varying intercept for each of the 858 topics entails at least two benefits. The random intercept allows me to quantify the within-topic path dependency in funding, by returning the average within-topic effect in a given year of different funding levels in 1-5 years prior. It takes into account that the amount of funding to a topic is measured repeatedly. Moreover, it gives an estimate of the variation in funding path dependency for all topics. The model is specified with the stochastic component

$$
F_{i t} \sim \operatorname{Hamma}\left(\pi_{i t}, \mu_{i t}, \theta_{i}\right)
$$

Where $F_{i t}$ denotes the amount of funding, for a topic $i$ in year $t . \pi_{i t}$ is the probability of zero funding for a topic in a given year, and $\mu_{i t}, \theta_{i}$ describes the distribution of non-zero funding

Then the systematic component can be given as

$$
\begin{gathered}
F_{i t}=\left\{\begin{array}{c}
\pi_{i t} \text { if } F_{i t}=0 \\
\left(1-\pi_{i t}\right) \operatorname{Gamma}\left(\mu_{i t}, \theta_{i}\right) \text { if } y>0
\end{array}\right. \\
\log \frac{\pi_{i t}}{1-\pi_{i t}}=\beta_{0 i t}+\lambda_{i}+\sum_{k=1}^{5} \beta_{k} F_{i j t-k} \\
\log \mu_{i t}=\beta_{0 i t}+\lambda_{i}+\sum_{k=1}^{5} \beta_{k} F_{i j t-k}
\end{gathered}
$$

With $F_{i j t-k}$ being a vector of lagged funding levels such that $k=\{1,2,3,4,5\}, \beta_{k}$ are the fixed population-averaged/fixed effects of previous funding, and $\lambda_{i}$ is the random intercept.

All five variables of lagged funding amounts were mean-normalised, divided by one standard deviation, and were assigned a Cauchy prior probability such that $\beta_{k} \sim \operatorname{Cauchy}(0,2.5)$, and the intercept, $\beta_{0 i t} \sim$ Cauchy $(0,20)$. In the supplementary material (Figure S6), I report 12 models with alternative prior specifications. These models produce similar results. All posterior distributions were estimated empirically by Hamiltonian Markov Chain Monte Carlo (HMC) using R (ver. 3.6.2) and the package 'brms' (Bürkner 2017, 2018) For all models, I assigned a 2,000 burn-in and 8,000 iterations for the sampler, using two chains, an acceptance rate of 0.9 (adapt delta), and a max tree depth of 20. The visualisations of the descriptives and posterior distributions were made with the R packages 'tidybayes' (Kay 2019) and 'ggplot2' (Wickham 2016). 
A

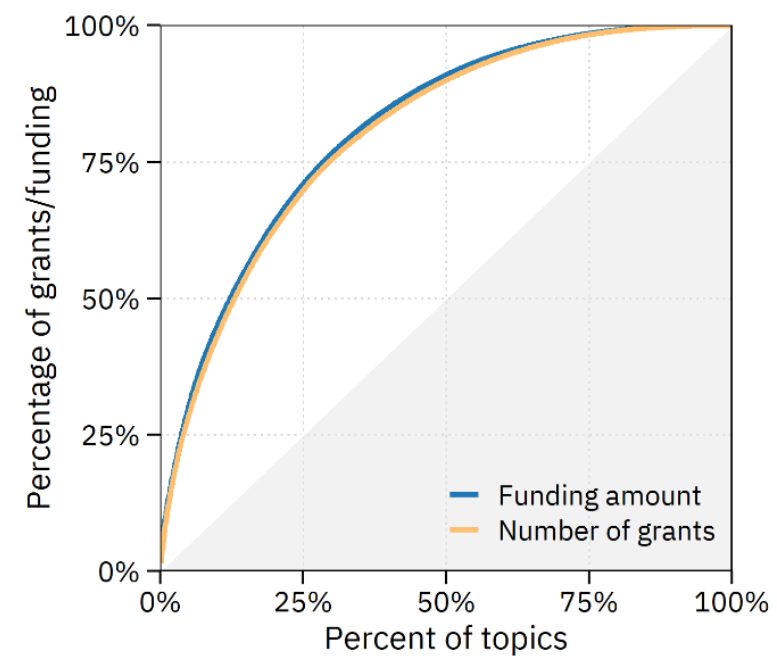

B

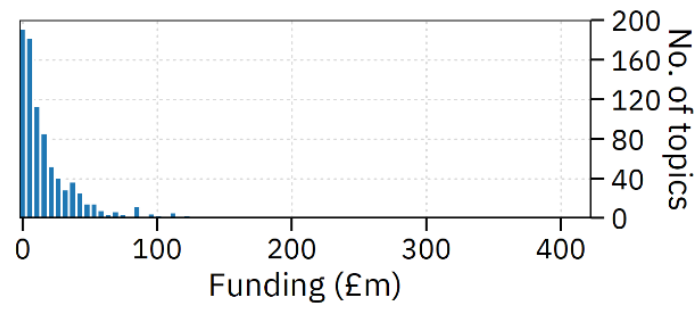

C

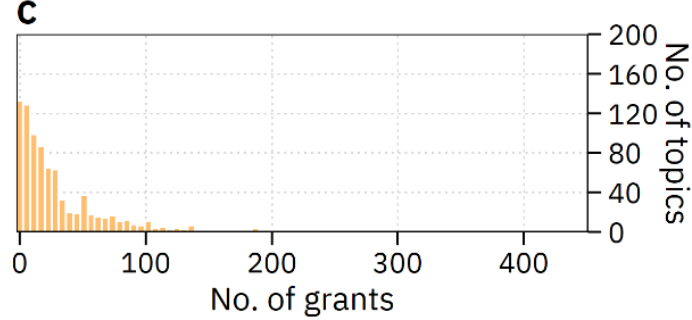

Figure 2. Distribution of funding to topics, 2005-2017. (A) Cumulative distribution of funding and grants to 858 topics. (B) Distribution of funding amounts. (C) Distribution of number of grants.

\section{Results}

I present the results of the analysis in two consecutive sections. The first section focus on the issue of skewness and concentration of funding to research topics. I present a range of descriptive evidence for the full time period (2006-2017) and show how concentration has been stable over time. The second section focus on the dynamic interpretation of cumulative advantage, and presents both descriptive and model-based evidence of stasis or path dependency.

\subsection{The skewness of topic prominence in research funding}

In order to investigate the concentration or dispersal across different research topics, I calculated the cumulative funding to each of the 858 topics across the entire period. Figure 2 shows the distributions of both the cumulative grant amounts, and the cumulative number of grants per topic. Figure 2.A plots the cumulative distributions across all topics through a Lorenz curve. Complete equality of funding and grants would entail the two distributions tracking the 45-degree diagonal. Instead, I find a high degree of concentration with $10 \%$ of topics amassing $44.8 \%$ of funding and $43 \%$ of grants, or $25 \%$ of topics receiving $70 \%$ of both funding amounts and grant numbers. Figure 2.B and 2.C shows the two distributions of both funding amounts and grant numbers in absolute terms. In the supplementary material, Figure S2 shows Lorenz curves for each of the UK research councils included here. The concentration of funds in each council is even more skewed than across all councils. Consequently, the skewness of funding does not seem to be an artifact of the grant sample I am using. 

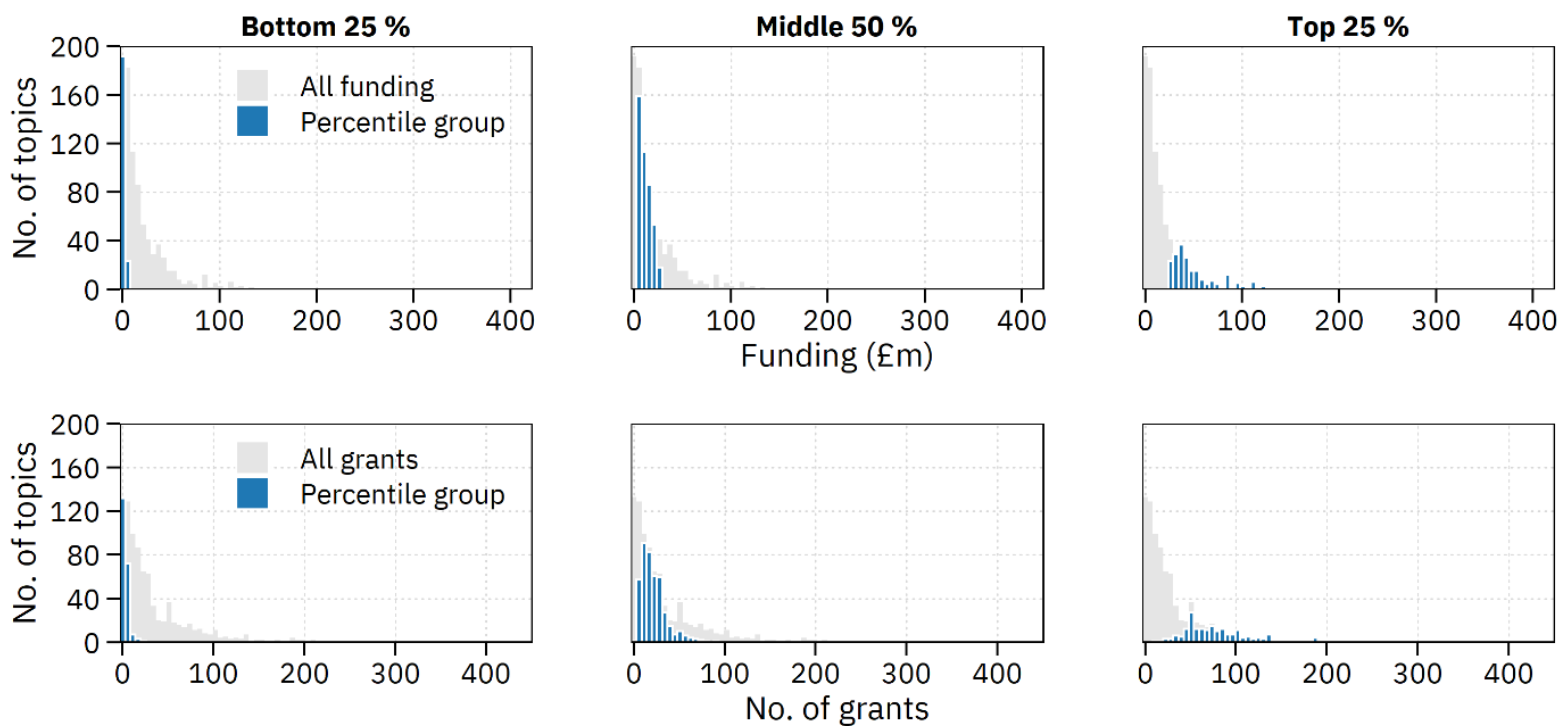

Figure 3. Distribution of funding and grants in percentile groups. Percentile groups are based on the distribution of funding amounts.

Across the more than 30,000 grants, the research councils distributed close to $£ 18$ billion during the entire period, and 85 of the 858 research topics accumulated around $£ 8.69$ billion of the entire sum, while 214 topics received almost $£ 12.8$ billion. One of the driving factors behind the concentration of funds in a small number of topics may be the differences in monetary demands across fields of research. Derek de Solla Price first noted that the cost of conducting research had generally increased rapidly following the Second World War (Price 1963). However, these costs are likely not evenly spread out. Some research questions are more expensive to answer than others. In the case of the distribution of funding, this might entail that the most well-off topics do not necessarily accumulate more grants, but just larger ones. Figure 3 shows that this is not the case. The top $25 \%$ of funded topics are funded through a combination of many smaller grants and a few bigger grants.

Besides the tendency for high degrees of funding concentration, a related concern has been that changing research funding systems increasingly facilitate higher degrees of concentration. Generally, analyses have corroborated these concerns with respect to concentration of funds to individual researchers or institutions (Katz and Matter 2019; Ma, Mondragón, and Latora 2015). To investigate a possible increasing concentration, Figure 4 plots three indicators of concentration across time. Figure 4.A shows that the proportion of funding awarded to the top $25 \%$ of topics is actually more concentrated on a year-by-year basis than for the entire period. 

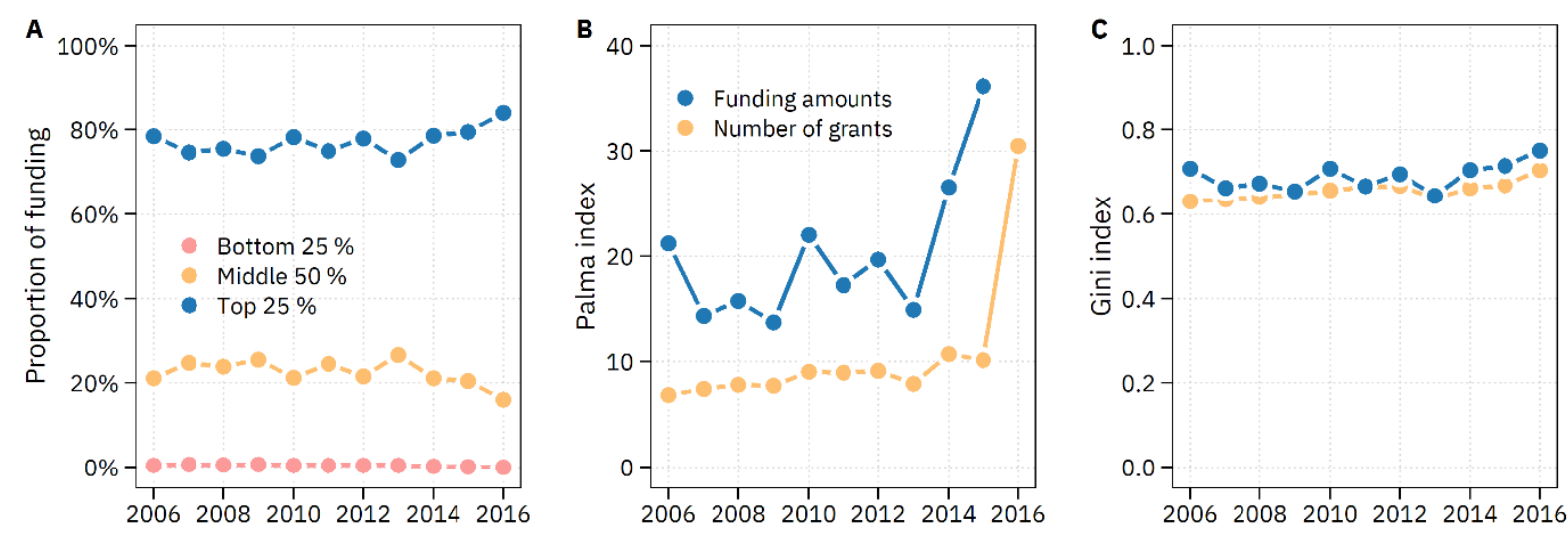

Figure 4. Concentration of funding and grants, 2006-2016. (A) Proportion of funding amounts in three percentile groups. (B) Palma index of funding amounts and grant numbers. (C) Gini index of funding amounts and grant numbers.

The top group of topics consistently attract close to $80 \%$ of all funding, while the middle $50 \%$ accounts for close to $20 \%$. The skewness of the yearly funding distributions are also reflected in the two inequality measures. The Palma index in Figure 4.B show some fluctuation, but the top $10 \%$ of topics attract, on average, 20 times the amount of funding awarded to the bottom $40 \%$. Similarly, the Gini coefficient in Figure 4.C shows substantial concentration with a value well above 0.6. In sum, I find a stark concentration of research funding in a few research topics. While this may indicate at least some cumulative advantage for certain topics, it does not imply that the same research topics continuously accumulate more funding because of early success or path dependency. To answer this question more precisely, we need to look into the funding accumulation within topics, and not just across them. This latter point is the focus on the following section.

\subsection{Stasis and path dependency for funded topics}

The question of how much, if at all, individual topics accumulate funding unevenly over time is important in shedding light on cumulative advantage in research funding. This is essentially the central mechanism of Merton's Matthew Effect. Recognition is not just distributed in a skewed fashion, but also deepens as time goes by. It is also a central finding in much of the literature on topic concentration in research literature (Foster, Rzhetsky, and Evans 2015; Rzhetsky et al. 2015; Stoeger et al. 2018). Topics that proved important early are more likely to be funded and investigated later. To shed light on this mechanism, I make use of the time dynamics in the dataset of grants. In Figure 5, I calculate the funding percentile of each topic when first funded in the period 2006-2017. I group each topic into four percentile bins (Top $25 \%$, 50-75 \%, 25-50\%, and Bottom $25 \%$ ) of 214- 215 topics each, and calculate each topic's funding percentile in the following five years. 

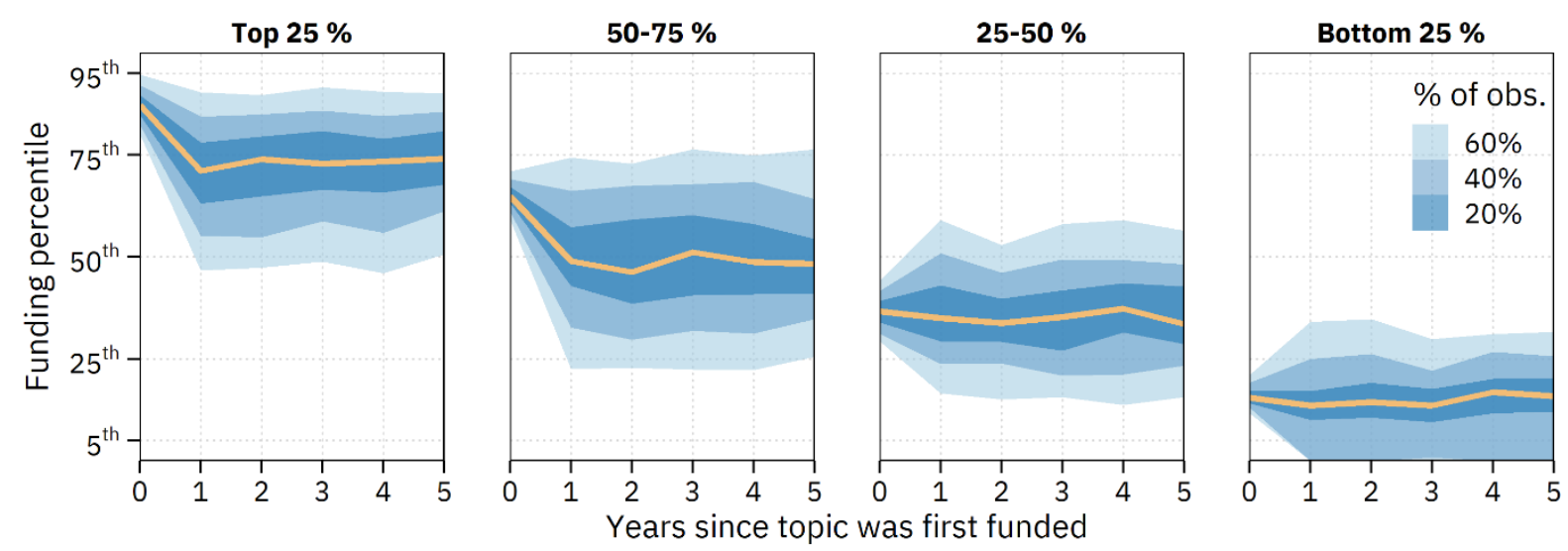

Figure 5. Subsequent funding percentiles for topics. Groups correspond to $25 \%$ increments of the funding distribution when topics were first funded.

The central tendency in the figure is the median funding rank of each of the four groups, while the shaded areas include $20 \%, 40 \%$, and $60 \%$ of the percentile distribution. The topics entering the funding distribution within the top $25 \%$ have a tendency to stay within the top in subsequent years. The median funding rank of this group is the $71^{\text {st }}$ percentile one year after, and increases slightly to the $73^{\text {rd }}$ percentile in each of the subsequent four years. Conversely, the group of topics starting out in the bottom $25 \%$ tend to hover around the $15^{\text {th }}$ percentile in the following five years. Figure S4 in the supplementary material repeats this analysis for groups of $10 \%$ (with 85 topics in each), where the top $10 \%$ are generally within the $85^{\text {th }}$ funding percentile in the years after first being funded. Figure S5 shows that, within funding councils, the stasis of funding distributions is also evident within the biosciences (BBSRC), engineering and physics (EPSRC), and medical sciences (MRC); the three areas most likely to be covered in the Web of Science. Figure 6 shows the relative proportion of funding for each of the four groups each year after first funded. The top $25 \%$-group receive $66-71 \%$ of the total funding amounts in the five years succeeding, while the $50-75 \%$ group receive $22-25 \%$ of the yearly funding. These results at least indicate some degree of path dependency and stasis in what research topics are prioritised in the competition for funding. The top group of topics tend to remain within the top of the funding distribution, while groups of topics with lower initial funding tend to remain in the lower percentiles of later funding distributions. However, Figure 5 also reveals a rather wide spread in what funding percentile the well-funded topics fall in to in the years after first entering the dataset. For the top $25 \%$ of topics, $60 \%$ span the $45-90^{\text {th }}$ percentile interval of funding in the years after. For the top $10 \%$ this interval is approximately the $78-89^{\text {th }}$ percentiles. The spread of subsequent funding percentiles is less pronounced in the bottom of the distribution. $60 \%$ of the topics in the bottom $10 \%$ of initial funding, never rise to more than the first $25^{\text {th }}$ funding percentile. This is not to say that topics with low initial 
funding never become more well-off. Some topics in the bottom $10 \%$ actually end up around the $45^{\text {th }}$ funding percentile.

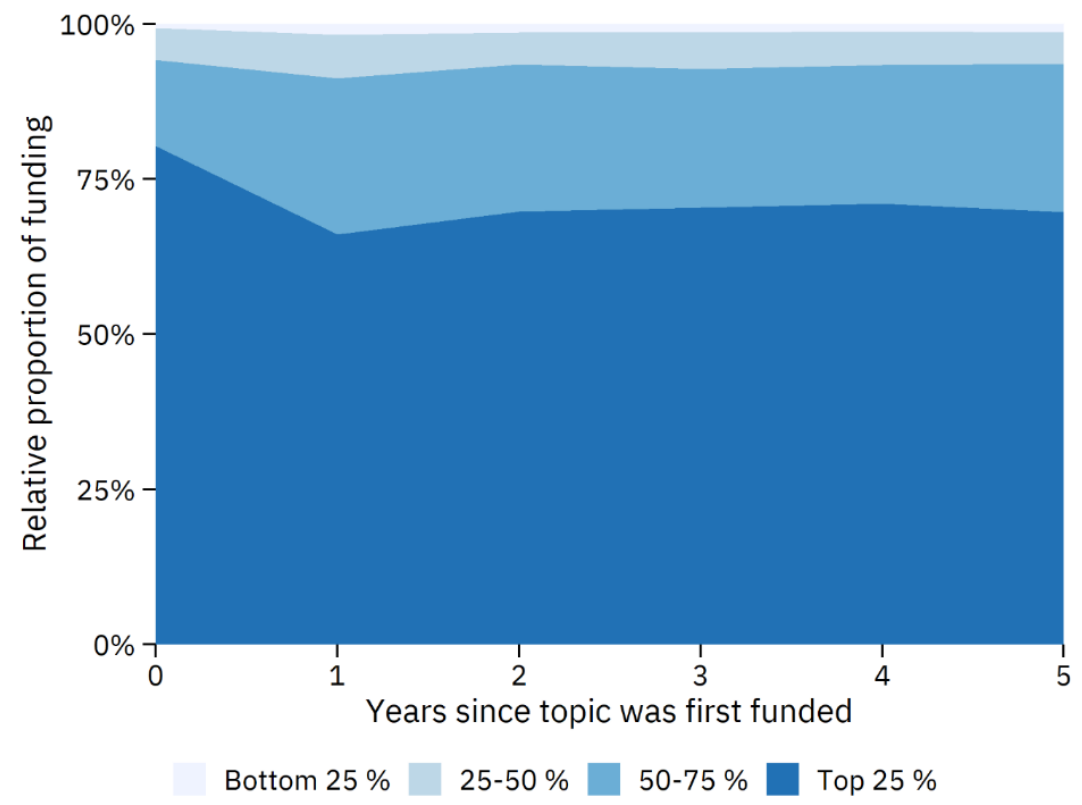

Figure 6. Relative proportion of funding for topics. Groups correspond to different parts of the funding distribution when topics were first funded.

My results seem to affirm that some cumulative advantages for topics do take place in the competition for funding, but it is by no mean a deterministic fact that the rich always get richer, while the poor stay poor. However, the topics in the absolute bottom or top of the distribution tend to stay within the bottom- or top half of later grant distributions.

These tendencies are factors of different groups of topics. To zoom in on how individual topics accumulate funding, I end by presenting results from the statistical model of path dependency presented above. The model relates the amount of funding a topic receives to the past levels of funding for that topic. Figure 7 plots the population-average ("fixed" effects) amount of funding for a topic, based on the amount of funding in the previous one to five years. Each of the five panels show the expected mean amount of funding at different levels of previous funding. I show the expected mean funding in a given year for 0 to 20 million pounds of in previous years, as this approximately incorporates five standard deviations of previous funding (for all time lags). Based on increasing amounts of previous funding in one to four years, the model indicates a slight tendency for cumulative advantages. A level of previous funding of about five million pounds in one to four years prior approximately translates into an average of around 1 million pounds of funding in a given year. Based on the model, this result seem to be quite robust. Figure 7 shows the population average, with $95 \%$ credible intervals, meaning that a premium of 
five million pounds of prior funding is highly likely to fall within a range of $£ 900,000$ to $£ 1,600,000$. Another noteworthy finding is the apparent curvelinear relationship between current and past funding.
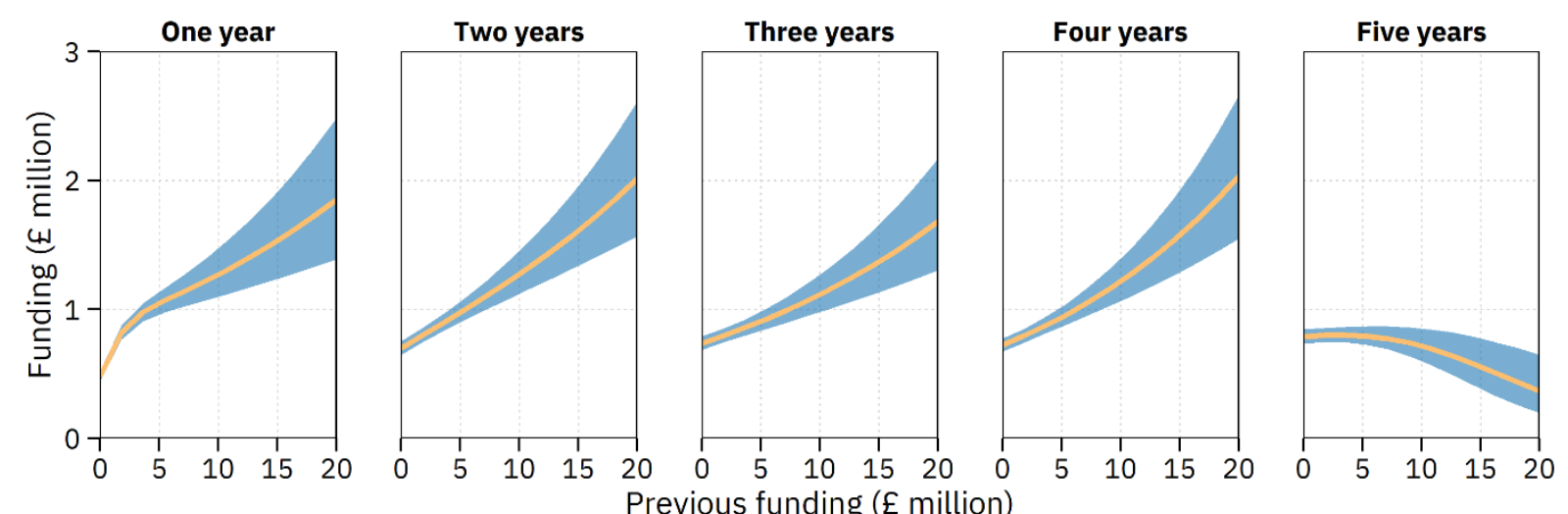

Figure 7. Average funding amounts for topics conditional on previous funding amounts. Each panel shows the population average posterior fit for previous funding one to five years before. Posterior fits based on model 3 in Table S2 with $95 \%$ credible intervals.

One example is the relationship between current funding amounts and funding amounts two years prior. Here, the difference between no previous funding and five millions pounds of previous funding amounts to a difference in current funding around $£ 400,000$. Looking at the difference between $£ 15$ million and $£ 20$ million in previous funding, my model indicates a difference in current funding of almost $£ 600,000$. This increasing marginal return to previous funding is more pronounced for higher levels. Figure S7 is identical to Figure 7, but with the average current funding estimated for previous funding levels of 50 to 90 million pounds. A difference in previous funding (two years prior) between 50 and 55 million pounds corresponds to a difference of around two million pounds. However, as the amount of previous funding increases so does the width of the credible intervals. For previous funding of 50 million pounds two years before, the premium could be as low as four million or as high as 15 million pounds.

Lastly, we should note the different effect funding five years prior seem to have on current funding levels. Instead of an increasing return to previous funding, past funding levels seem to matter little to current funding, or even be negatively related. For lower levels of previous funding (0-5 million pounds) a change from 0 to 5.4 million only increases current funding by $£ 3,280$, while a 5.4 million pound difference in previous funding at 21 million vs. 16 million translates into a decrease in current funding by $£ 196,114$.

\section{Discussion and conclusion}


The impact of competitively awarded research funding on the direction and content of science and the science system have attracted much attention. One persistent fear have been that researchers' increased reliance on competitive grant money leads to a narrowing of topics studied. Yet, we actually have little empirical evidence on how funding is distributed across different research topics and whether funding distributions are a prone to a cumulative advantage, which may exacerbate this narrowing. Here, I have tried to ascertain whether some research topics actually experience a form of cumulative advantage in funding, but I would argue that a definite answer is not yet warranted. Heeding the cautious words of earlier investigations of cumulative advantage in science: "Although the basic idea of cumulative advantage is quite simple, it is actually a rather complex hypothesis involving numerous causal links organized into several feedback loops. As a consequence, it is difficult to specify just what empirical findings would disconfirm the hypothesis" (Allison, Long, and Krauze 1982, 622). My analyses do however indicate that some of the proposed components of a Matthew effect in funding of topics are present. Firstly, the distribution of funding to different research areas are highly skewed with $25 \%$ of research topics amassing $70 \%$ of all funding. This concentration are not simply due to a few topics being awarded a few large grants, it recurs across very different research fields, and is stable throughout the period. Secondly, stasis or path dependency is a central tendency in the distribution of funding across time, but with some exceptions. The top $10 \%$ and top $25 \%$ of topics in terms of funding tend to stay within the 85th and 75th funding percentile, while the topics in the bottom $25 \%$ of the distribution generally remain in the bottom group. However, topics just outside of the top $25 \%$ do occasionally end up in the top of the distribution, so path dependency seems to primarily affect the very top or bottom. Lastly, there is a slight premium and increasing return to funding for a topic based on previous funding. This is congruent with earlier findings that also show past funding levels to be the best predictor of future funding levels (Klavans and Boyack 2017a). In this paper, I show that this relationship may be one of increasing marginal returns, with small amounts of prior funding leading to very small future funding levels, while very large amounts of prior funding yielding much large future returns. However, the amount of funding in prior and current time periods are not 1:1, as the predicted amount of funding is substantially lower than the previous levels. Figure S7 shows the difference between prior funding and predicted funding for all time lags. For one to four year lags of funding, previous funding needs to surpass 100 million pounds before the expected current funding amounts are larger.

Why some research topics seem to garner more attention than others is likely a question with multiple answers, but the results at least indicate that funding allocations are one piece of the puzzle. However, the analyses I have presented cannot discern the driving factors of cumulative advantage in science funding. I want to highlight two possible types of explanations. One is the different drivers of cumulative advantage that are built-in to scientific systems. These can broadly be thought of as the prestige effects I alluded to above, which have been the main focus of earlier investigations of funding concentration. Success in academia is often assed through past achievements, such as publications, grants, or awards. Many different studies show a concentration of funds on individual researchers or 
institutions, and document how certain researchers and research organisations continue to accumulate the bulk of all funding. This concentration is likely to spill-over into the type of research conducted. If few researchers and organisations receive the majority of funding, and also research a narrow set of topics, the concentration of funding in one domain may impact the other. Likewise, the past success of a research topic in the form of early breakthroughs, a well-developed research literature, and proved applicability, or many citations, may spur further funding of such topics because they appear promising.

Another type of drivers are systemic and caused by external pressures. This could be funders focusing on a more limited portfolio of topics they hope will lead to immediate inventions or solutions to societal problems. This is often argued to be the case in biomedicine, where translational research with close links to medical practice is prioritised over more fundamental research (Alberts et al. 2014, 5774). It may also be that researchers foresee what topics might be deemed "fundable" and self-select into research avenues they are more likely to obtain funding for (Gläser and Laudel 2016; Laudel and Gläser 2014). More worrying, topic selection and funder priorities may suppress specific topics as some researchers experience less funding success with novel research (Wang, Lee, and Walsh 2018). Evidence from the NIH also suggests that reviewers favour certain topics even if topics with lower success are just as or more cited than high-success topics (Hoppe et al. 2019).

The results presented here could be used as an opportunity to discuss how research funding systems should be organised. Complete parity of funding for all topics is not, and should not, be a goal in itself, but the persistent skew and stasis in what topics are funded should indicate that some measures to ensure more diversity is warranted. 


\section{References}

Alberts, B., M. W. Kirschner, S. Tilghman, and H. Varmus. 2014. "Rescuing US Biomedical Research from Its Systemic Flaws." Proceedings of the National Academy of Sciences 111(16): 5773-77.

Allison, Paul D., J. Scott Long, and Tad K. Krauze. 1982. "Cumulative Advantage and Inequality in Science.” American Sociological Review 47(5): 615-25.

Allison, Paul D., and John A. Stewart. 1974. "Productivity Differences among Scientists: Evidence for Accumulative Advantage.” American Sociological Review 39(4): 596-606.

Atkinson, Anthony B, Thomas Piketty, and Emmanuel Saez. 2011. "Top Incomes in the Long Run of History." Journal of Economic Literature 49(1): 3-71.

Best, Rachel Kahn. 2012. "Disease Politics and Medical Research Funding.” American Sociological Review 77(5): 780-803.

Bol, Thijs, Mathijs de Vaan, and Arnout van de Rijt. 2018. "The Matthew Effect in Science Funding." Proceedings of the National Academy of Sciences: 1-4.

Bromham, Lindell, Russell Dinnage, and Xia Hua. 2016. "Interdisciplinary Research Has Consistently Lower Funding Success.” Nature 534(7609): 684-87.

Bürkner, Paul-Christian. 2017. "Brms : An R Package for Bayesian Multilevel Models." Journal of Statistical Software 80(1): 1-28.

-—-. 2018. “Advanced Bayesian Multilevel Modeling with the R Package Brms.” The R Journal 10(1): 395-411.

Cobham, Alex, Lukas Schlögl, and Andy Sumner. 2016. "Inequality and the Tails: The Palma Proposition and Ratio.” Global Policy 7(1): 25-36.

Cole, Stephen, and Jonathan R. Cole. 1967. "Scientific Output and Recognition : A Study in the Operation of the Reward System in Science." American Sociological Review 32(3): 377-90.

Domenico, Manlio De, Elisa Omodei, and Alex Arenas. 2016. "Quantifying the Diaspora of Knowledge in the Last Century." Applied Network Science 1(15): 1-13.

Ebadi, Ashkan, and Andrea Schiffauerova. 2015. "How to Receive More Funding for Your Research? Get Connected to the Right People." PLoS ONE 10(7): 1-19.

Evans, James A., Jae Mahn Shim, and John P.A. Ioannidis. 2014. "Attention to Local Health Burden and the Global Disparity of Health Research.” PLoS ONE 9(4): 1-9.

Fang, Ferric C, and Arturo Casadevall. 2016. "Research Funding: The Case for a Modified Lottery." mBio 7(2): e00694-16. 
Fortin, Jean Michel, and David J. Currie. 2013. "Big Science vs. Little Science: How Scientific Impact Scales with Funding." PLoS ONE 8(6).

Fortunato, Santo et al. 2018. "Science of Science." Science 359(6379).

Foster, Jacob G., Andrey Rzhetsky, and James A. Evans. 2015. 'Tradition and Innovation in Scientists' Research Strategies.” American Sociological Review 80(5): 875-908.

Goldstone, Jack A. 1979. "A Deductive Explanation of the Matthew Effect in Science.” Social Studies of Science 9(3): 385-91.

Gross, Cary, Gerard Anderson, and Neil Powe. 1999. "The Relation between Funding by the National Institutes of Health and the Burden of Disease.” New England Journal of Medicine 340(24): 1881-87.

Gross, Kevin, and Carl T. Bergstrom. 2018. "Contest Models Highlight Inherent Inefficiencies of Scientific Funding Competitions.” PLoS Biology 17(1): e3000065.

Hagstrom, Warren O. 1974. “Competition in Science.” American Sociological Review 39(1): 1-18.

Hargens, Lowell L, Nicholas C Mullins, and Pamela K Hecht. 1980. "Research Areas and Stratification Processes in Science." Social Studies of Science 10: 55-74.

Head, Michael G. et al. 2013. "UK Investments in Global Infectious Disease Research 1997-2010: A Case Study.” The Lancet Infectious Diseases 13(1): 55-64.

-_- 2016. "Research Investments in Global Health: A Systematic Analysis of UK Infectious Disease Research Funding and Global Health Metrics, 1997-2013.” EBioMedicine 3: 180-90.

Head, Michael G, Joseph R Fitchett, David AJ Moore, and Rifat Atun. 2015. "Systematic Analysis of Funding Awarded to Institutions in the United Kingdom for Infectious Disease Research, 19972010." JRSM Open 6(3): 205427041557705.

Hoonlor, Apirak, Boleslaw K. Szymanski, and Mohammed J. Zaki. 2013. "Trends in Computer Science Research." Communications of the ACM 56(10): 74.

Jacob, Brian A., and Lars Lefgren. 2011. "The Impact of Research Grant Funding on Scientific Productivity.” Journal of Public Economics 95(9-10): 1168-77.

Jia, Tao, Dashun Wang, and Boleslaw K. Szymanski. 2017. "Quantifying Patterns of Research-Interest Evolution.” Nature Human Behaviour 1(4): 1-7.

Jones, Richard, and James Wilsdon. 2018. The Biomedical Bubble: Why UK Research and Innovation Needs a Greater Diversity of Priorities, Politics, Places and People. London: Nesta.

Katz, Yarden, and Ulrich Matter. 2017. Berkman Klein Center for Internet \& Society Research Publication On the Biomedical Elite: Inequality and Stasis in Scientific Knowledge Production.

-_-. 2019. "Metrics of Inequality: The Concentration of Resources in the U .S. Biomedical Elite." 
Science as Culture 0(0): 1-28.

Kay, Matthew. 2019. “Tidybayes: Tidy Data and Geoms for Bayesian Models.”

Klavans, Richard, and Kevin W. Boyack. 2017a. "Research Portfolio Analysis and Topic Prominence." Journal of Informetrics 11(4): 1158-74.

--_. 2017b. "The Research Focus of Nations: Economic vs. Altruistic Motivations." PLoS ONE 12(1): e0169383.

Larivière, Vincent, Benoit Macaluso, Éric Archambault, and Yves Gingras. 2010. "Which Scientific Elites? On the Concentration of Research Funds, Publications and Citations." Research Evaluation 19(1): 45-53.

Lerchenmueller, Marc J., and Olav Sorenson. 2018. "The Gender Gap in Early Career Transitions in the Life Sciences." Research Policy 47(6): 1007-17.

Long, J. Scott. 1978. "Productivity and Academic Position in the Scientific Career." American Sociological Review 43(6): 889-908.

Ma, Athen, Raúl J. Mondragón, and Vito Latora. 2015. "Anatomy of Funded Research in Science." Proceedings of the National Academy of Sciences 112(48): 14760-65.

Manton, Kenneth G. et al. 2009. "NIH Funding Trajectories and Their Correlations with US Health Dynamics from 1950 to 2004.” Proceedings of the National Academy of Sciences 106(27): 10981-86.

Meager, Rachael. 2019. "Understanding the Average Impact of Microcredit Expansions: A Bayesian Hierarchical Analysis of Seven Randomized Experiments." American Economic Journal: Applied Economics 11(1): 57-91.

Merton, Robert K. 1968. “The Matthew Effect in Science.” Science 159(3810): 56-63.

- - . 1988. "The Matthew Effect in Science , II: Cumulative Advantage and the Symbolism of Intellectual Property." Isis 79(4): 606-23.

Merton, Robert K. 1957. "Priorities in Scientific Discovery: A Chapter in the Sociology of Science." American Sociological Review 22(6): 635-59.

Mongeon, Philippe, Christine Brodeur, Catherine Beaudry, and Vincent Lariviere. 2016. "Concentration of Research Funding Leads to Decreasing Marginal Returns." Research Evaluation 25(4): 396-404.

Nature. 2003. "Editorial: Research Funding: The Problem with Priorities.” Nature Materials 2(10): 639.

Nichols, Leah G. 2014. "A Topic Model Approach to Measuring Interdisciplinarity at the National Science Foundation.” Scientometrics 100(3): 741-54.

Peifer, Mark. 2017. “The Argument for Diversifying the NIH Grant Portfolio.” Molecular Biology of the 
Cell 28(22): 2935-40.

Polanyi, Michael. 1962. “The Republic of Science: Its Political and Economic Theory.” Minerva 1(1): 54-73.

Price, Derek J. de Solla. 1963. Little Science, Big Science. New York, NY: Columbia University Press.

Reskin, Barbara F. 1977. "Scientific Productivity and the Reward Structure of Science.” American Sociological Review 42(3): 491-504.

Rzhetsky, Andrey, Jacob G. Foster, Ian T. Foster, and James A. Evans. 2015. "Choosing Experiments to Accelerate Collective Discovery." Proceedings of the National Academy of Sciences 112(47): 14569-74.

Sampat, Bhaven N. 2012. "Mission-Oriented Biomedical Research at the NIH." Research Policy 41(10): 1729-41.

Sarewitz, Daniel, and Roger A. Pielke. 2007. "The Neglected Heart of Science Policy: Reconciling Supply of and Demand for Science." Environmental Science and Policy 10(1): 5-16.

Small, Henry, and Belver C. Griffith. 1974. "The Structure of Scientific Literatures I: Identifying and Graphing Specialties.” Science Studies 4(1): 17-40.

Stoeger, Thomas, Martin Gerlach, Richard I. Morimoto, and Luís A. Nunes Amaral. 2018. "LargeScale Investigation of the Reasons Why Potentially Important Genes Are Ignored." PLoS Biology 16(9): 1-25.

Traag, V A, L Waltman, and N J Van Eck. 2019. "From Louvain to Leiden : Guaranteeing WellConnected Communities." Scientific Reports 9(5233): 1-12.

Vaesen, Krist, and Joel Katzav. 2017. "How Much Would Each Researcher Receive If Competitive Government Research Funding Were Distributed Equally among Researchers?” PLoS ONE 12(9): e0183967.

Wahls, Wayne P. 2018a. "High Cost of Bias: Diminishing Marginal Returns on NIH Grant Funding to Institutions." bioRxiv.

-—-. 2018b. "The NIH Must Reduce Disparities in Funding to Maximize Its Return on Investments from Taxpayers." eLife 7: 1-9.

Waltman, Ludo, and Nees Jan Van Eck. 2013. “A Smart Local Moving Algorithm for Large-Scale Modularity-Based Community Detection.” European Physical Journal B 86(11): 471.

Waltman, Ludo, and Nees van Eck. 2012. "A New Methodology for Constructing a Publication-Level Classification System of Science.” Journal of the American Society for Information Science and Technology 63(12): 2378-96.

Wickham, Hadley. 2016. Ggplot2: Elegant Graphics for Data Analysis. New York, NY: Springer-Verlag. 
Yao, Lixia et al. 2015. "Health ROI as a Measure of Misalignment of Biomedical Needs and Resources." Nature Biotechnology 33(8): 807-11.

Yegros-Yegros, Alfredo, Robert Tjissen, Maria-Francisca Abad-Garcia, and Ismael Rafols. 2018. "Drug Research Priorities at Odds with Global Disease Toll.” Nature Index 16 March.

Zuckerman, Harriet. 1967. "Nobel Laureates in Science: Patterns of Productivity, Collaboration , and Authorship.” American Sociological Review 32(3): 391-403. 


\section{Supplementary materials}

Diversity or Disparity? The Concentration of Funded Research Topics in the United Kingdom 
A. Sample of grants used in analysis 


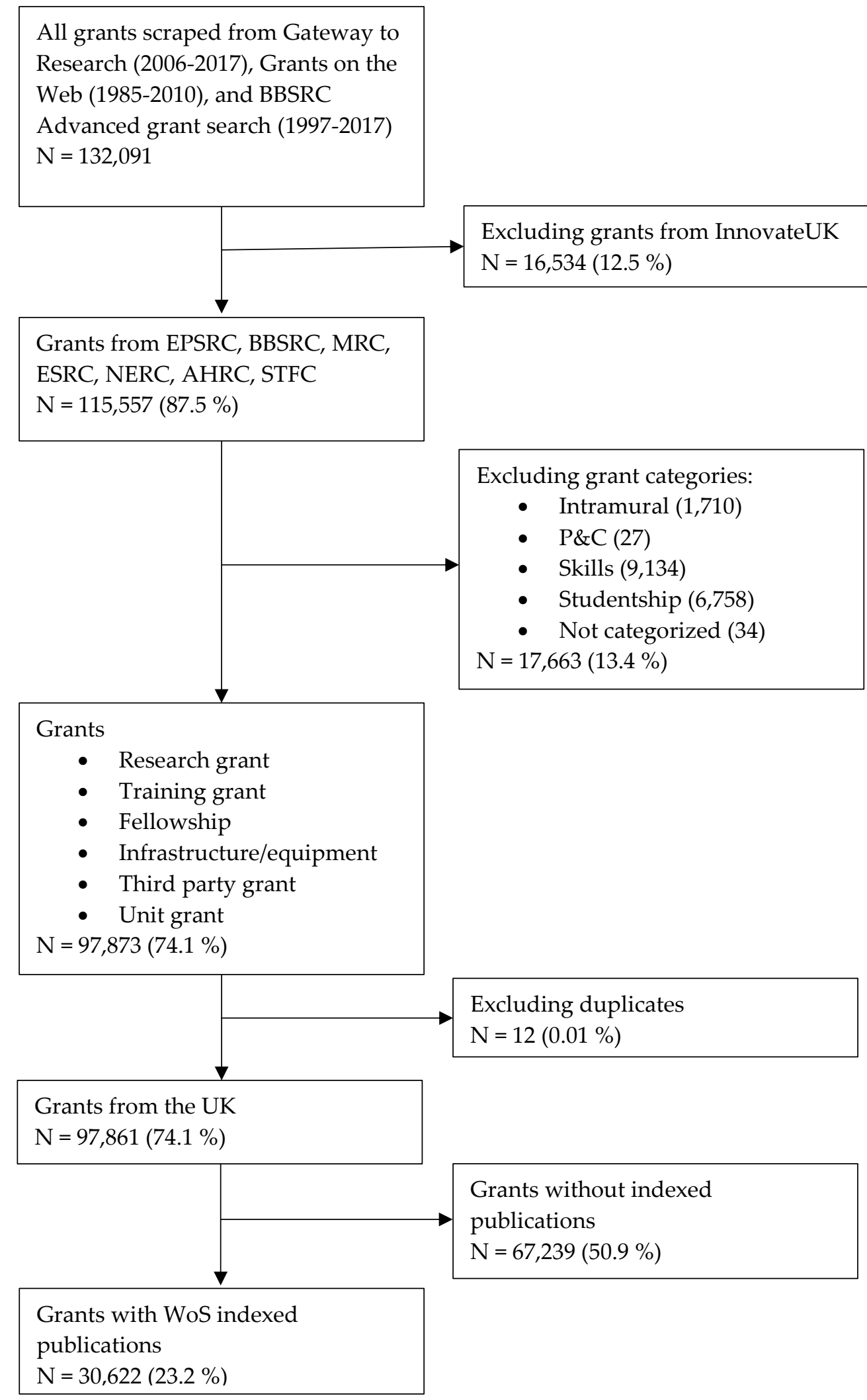

Figure S1. Final grant sample. 


\section{B. Additional analyses of skew}

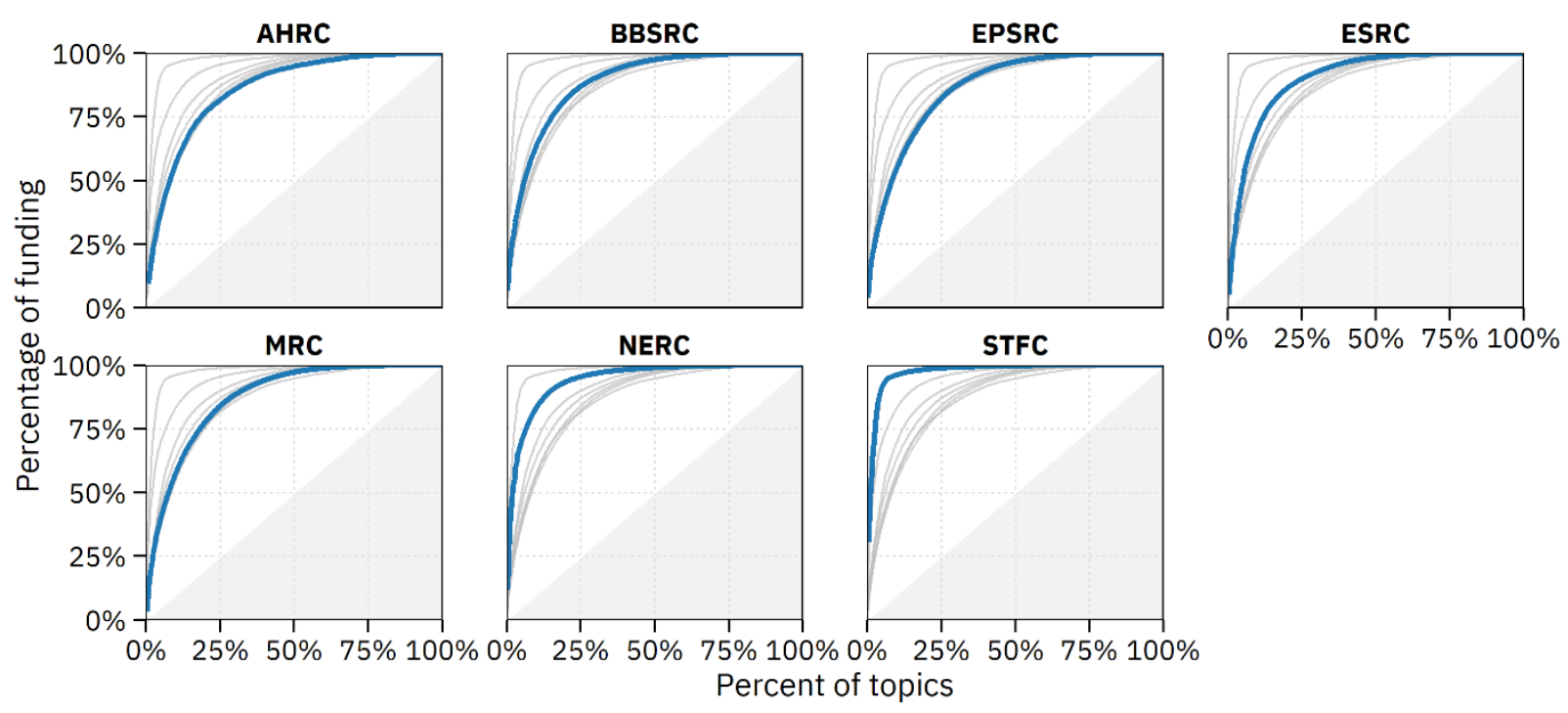

Figure S2. Cumulative distribution of funding amounts over topics for each research council.
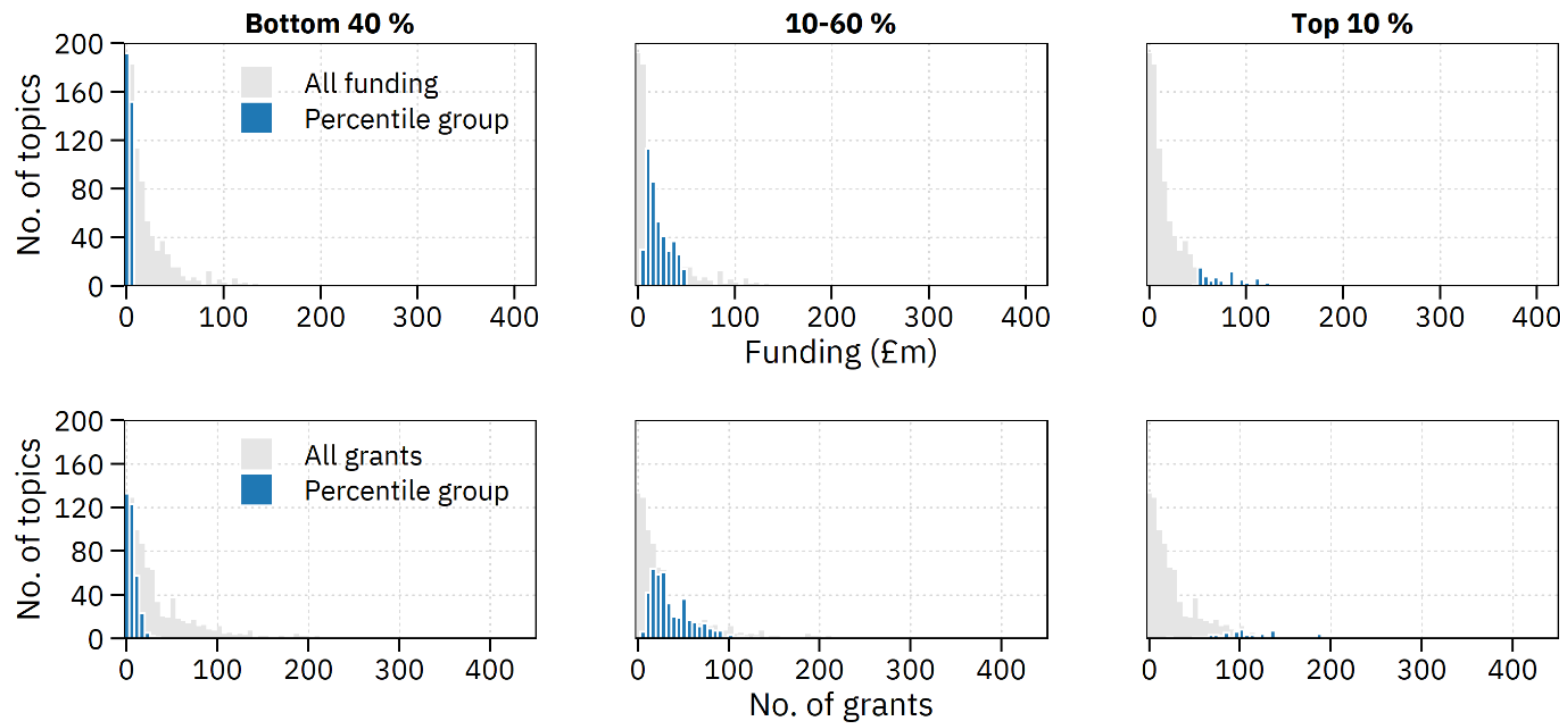

Figure S3. Distribution of funding and grants in percentile groups. Percentile groups are based on the distribution of funding amounts. 


\section{Additional analyses of stasis}

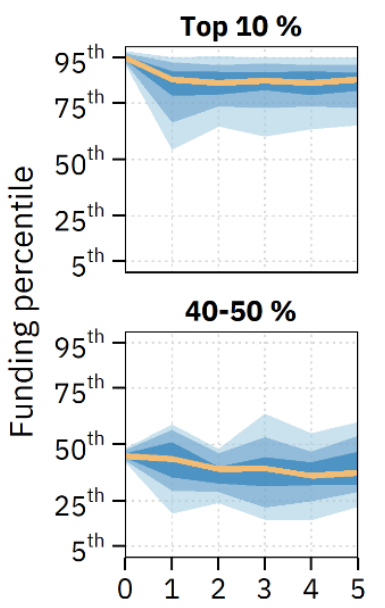

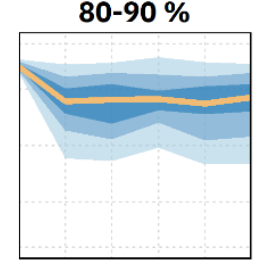

$30-40 \%$
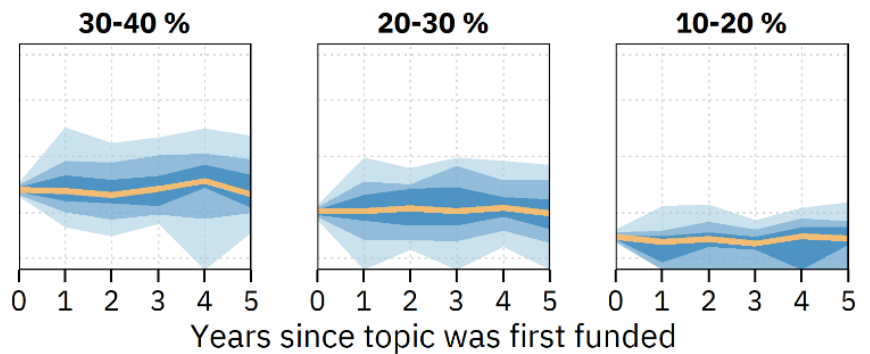

$60-70 \%$
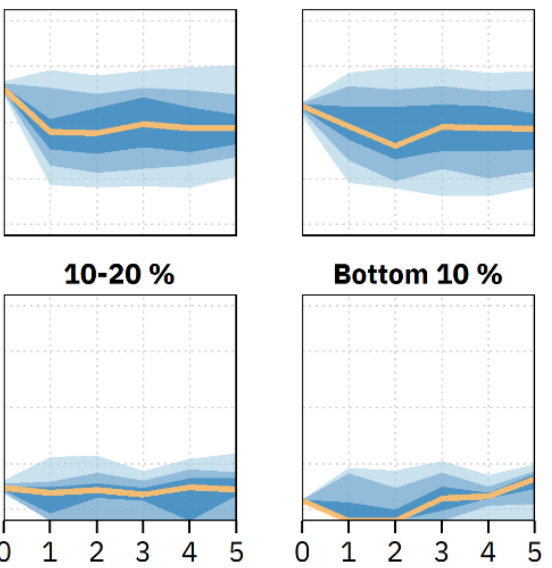

\begin{tabular}{l|l|l|l} 
\% of obs. & $60 \%$ & $40 \%$ & $20 \%$
\end{tabular}

Figure S4. Subsequent funding percentiles for topics. Groups correspond to $10 \%$ increments of the funding distribution when topics were first funded.
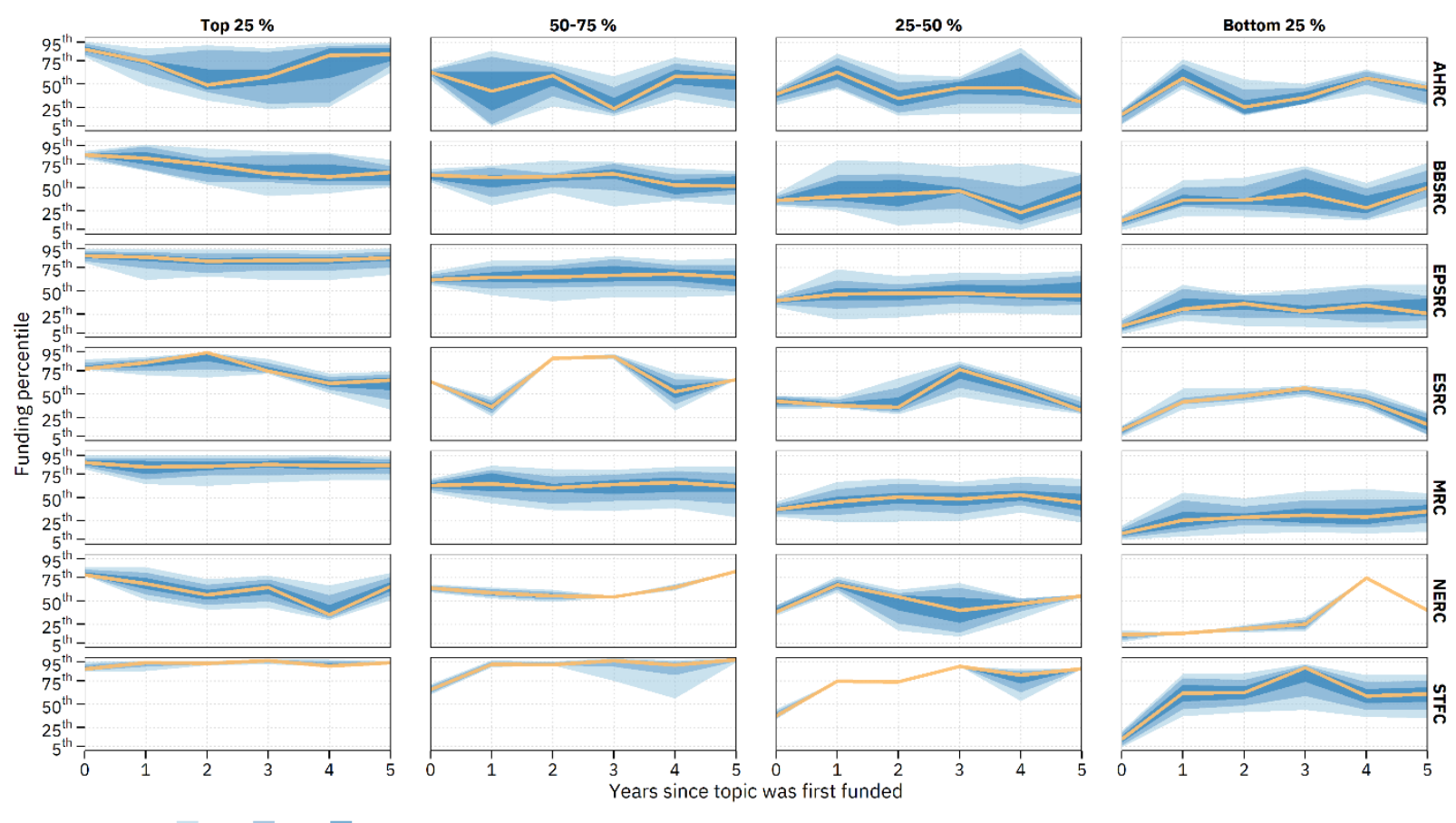

$\%$ of obs. $\square 60 \% \square 40 \% \square 20 \%$

Figure S5. Subsequent funding percentiles for topics in each research council. Groups correspond to $25 \%$ increments of the funding distribution when topics were first funded. 


\section{Regression models of cumulative advantage}

For the regression model of cumulative advantage, I specify a prior for both the effects of earlier funding, and the intercept. Priors help constrain the model to avoid fitting noise in the data. In essence, prior distributions of the parameters help regularise the estimates by introducing additional information about the likely parameter space, which constrains the model procedure through a reduction of variance (Meager 2019, 67). However, the lesser variance comes at a cost of higher bias compared to frequentist unbiased estimates. For all priors, I centre the prior distribution around zero to reflect an initial scepticism towards an effect, but vary the type of prior distribution and the scale parameter to allow for more or less regularisation. For all models, I standardised the input variables, the lagged funding levels, by centering them on the average and dividing by one standard deviation. This seemed reasonable, as the lagged funding levels are distributed in a very skewed fashion, and putting the variables on a standardized scale reduces the variance in them and facilitate easier convergence of the model. The raw coefficients from the standardised input variables are presented in Table S1, but in all figures presented here and in the main text, I converted the standardised estimates back to the orgininal scale. I show that the posterior inferences derived from the model presented in the main text is robust to a reasonable alternative prior specification. Table S1 gives the twelve prior specifications, and Figure S6 shows the main coefficients for each model, with $95 \%$ credible intervals. Table S2 provides the posterior means and quantiles for the model reported in the main text.

Table S1. Prior robustness specifications

\begin{tabular}{cc}
\hline Prior specification number & Priors \\
\hline 1 & $\beta_{k} \sim N(0,3), \beta_{0 i t} \sim N(0,20)$ \\
2 & $\beta_{k} \sim N(0,5), \beta_{0 i t} \sim N(0,20)$ \\
3 & $\beta_{k} \sim N(0,7), \beta_{0 i t} \sim N(0,20)$ \\
4 & $\beta_{k} \sim N(0,3), \beta_{0 i t} \sim N(0,40)$ \\
5 & $\beta_{k} \sim N(0,5), \beta_{0 i t} \sim N(0,60)$ \\
6 & $\beta_{k} \sim N(0,7), \beta_{0 i t} \sim N(0,80)$ \\
7 & $\beta_{k} \sim \operatorname{Cauchy}(0,5), \beta_{0 i t} \sim \operatorname{Cauchy}(0,20)$ \\
8 & $\beta_{k} \sim \operatorname{Cauchy}(0,7.5), \beta_{0 i t} \sim \operatorname{Cauchy}(0,20)$ \\
9 & $\beta_{k} \sim \operatorname{Cauchy}(0,10), \beta_{0 i t} \sim \operatorname{Cauchy}(0,20)$ \\
10 & $\beta_{k} \sim \operatorname{Cauchy}(0,5), \beta_{0 i t} \sim \operatorname{Cauchy}(0,40)$ \\
11 & $\beta_{k} \sim \operatorname{Cauchy}(0,7.5), \beta_{0 i t} \sim \operatorname{Cauchy}(0,60)$ \\
12 & $\beta_{k} \sim \operatorname{Cauchy}(0,10), \beta_{0 i t} \sim \operatorname{Cauchy}(0,80)$ \\
\hline
\end{tabular}



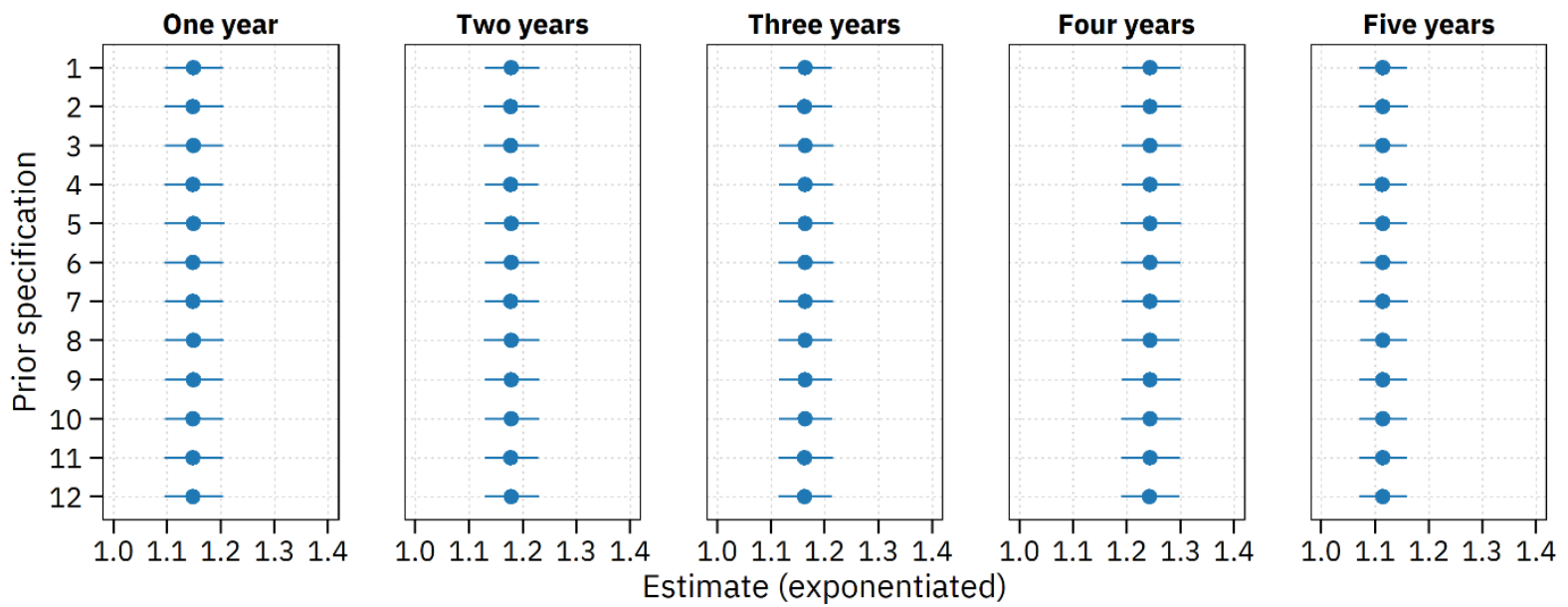

Figure S6. Posterior summary from twelve different models. Posterior medians with $95 \%$ credible intervals

Table S2. Bayesian model of path dependence in funding

\begin{tabular}{cccccc}
\hline \hline & Estimate & \multicolumn{3}{c}{ Posterior distribution quantiles } \\
\cline { 2 - 6 } & $\beta_{k}$ & $2.5^{\text {th }}$ & $25^{\text {th }}$ & $75^{\text {th }}$ & $95^{\text {th }}$ \\
\hline Predicting level of funding & & & & & \\
$\beta_{\text {0it }}$ & 13.72 & 13.65 & 13.70 & 13.74 & 13.78 \\
One year lag & 0.14 & 0.08 & 0.12 & 0.16 & 0.20 \\
Two years lag & 0.16 & 0.11 & 0.15 & 0.18 & 0.22 \\
Three years lag & 0.15 & 0.10 & 0.13 & 0.17 & 0.20 \\
Four years lag & 0.22 & 0.17 & 0.2 & 0.24 & 0.27 \\
Five years lag & 0.11 & 0.06 & 0.82 & 0.86 & 0.16 \\
Std. dev. of $\lambda_{i}$ & 0.84 & 0.77 & 0.82 & 0.86 & 0.91 \\
\hline Predicting no funding & & & & & \\
$\beta_{\text {0it }}$ & -1.78 & -1.89 & -1.82 & -1.74 & -1.67 \\
One year lag & -3.65 & -4.00 & -3.77 & -3.53 & -3.30 \\
Two years lag & -0.72 & -0.93 & -0.79 & -0.65 & -0.52 \\
Three years lag & -0.09 & -0.19 & -0.13 & -0.05 & 0.04 \\
Four years lag & 0.08 & -0.02 & 0.04 & 0.11 & 0.20 \\
Five years lag & 0.65 & 0.48 & 0.59 & 0.71 & 0.81
\end{tabular}


Notes: All estimates are on a standardised and mean-centered scale. Estimates of funding levels are on a log-scale, while estimates for predicting zero funding are on the logit scale.
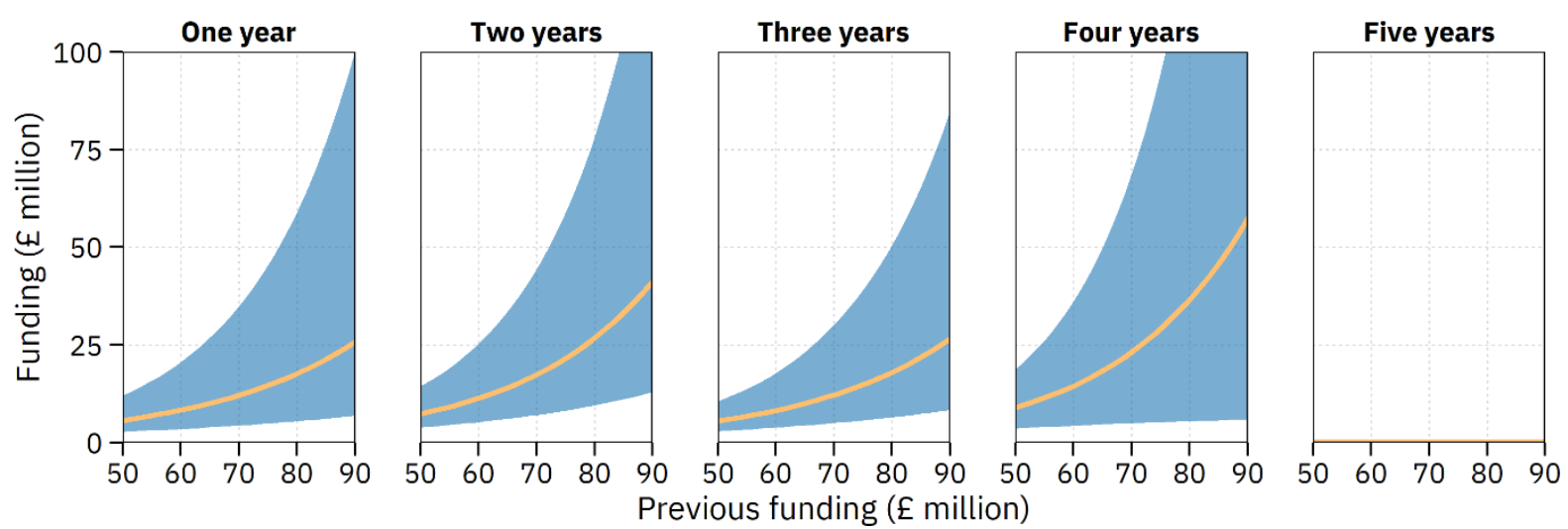

Figure S7. Average funding amounts for topics conditional on previous funding amounts.

Each panel shows the population average posterior fit for previous funding one to five years before. Posterior fits are based on model 3 in Table S1 with 95 \% credible intervals.
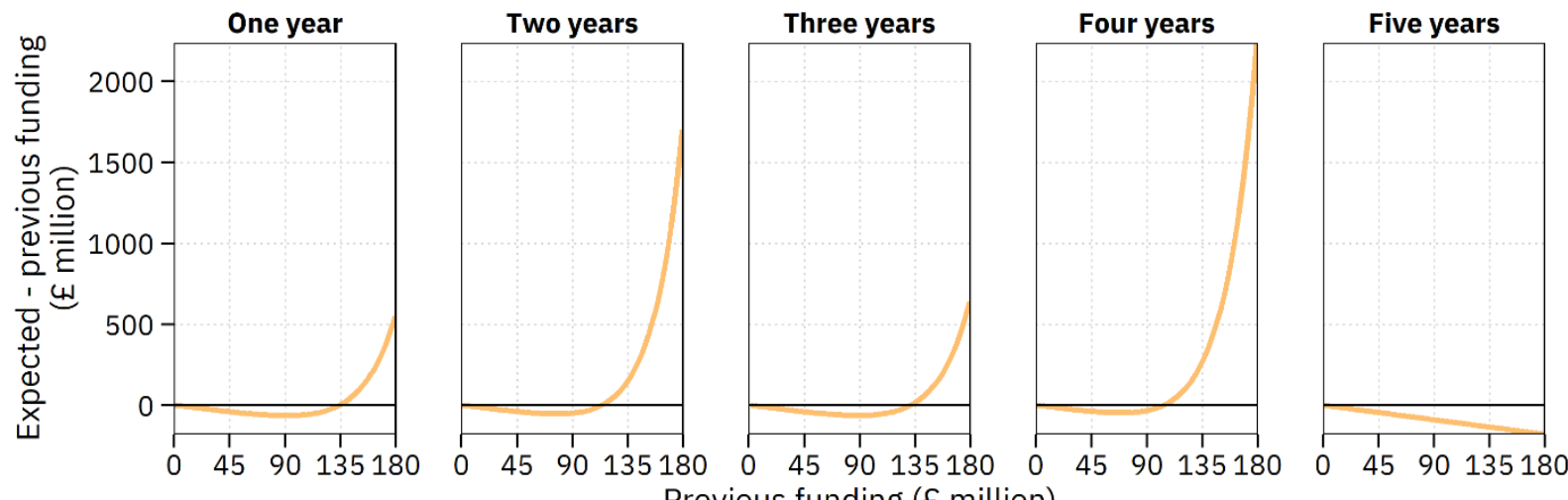

Figure S8. Expected mean funding amount minus previous funding amount. Each panel shows

the difference between the expected amount of funding in a given year (population average posterior fit) and previous funding one to five years before. Posterior fits are based on model 3 in Table S1 with $95 \%$ credible intervals. 